\title{
Métricas alternativas de periódicos da Ciência da Informação
}

\author{
Ednéia Silva Santos Rocha ${ }^{I}$ \\ http://orcid.org/0000-0003-1478-6828 \\ Márcia Regina da Silva ${ }^{I I}$
}

${ }^{I}$ Universidade de São Paulo, SP, Brasil.

Doutora em Política Científica e Tecnológica pela Universidade Estadual de Campinas (UNICAMP). Bibliotecária da Faculdade de Direito de Ribeirão Preto da Universidade de São Paulo (FDRP/USP).

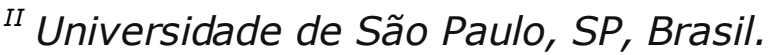

Doutora em Educação pela Universidade Federal de São Carlos (UFSCar). Coordenadora do Curso de Biblioteconomia, Ciências da Informação e da Documentação da Faculdade de Educação, Ciências e Letras de Ribeirão Preto.

\section{http://dx.doi.org/10.1590/1981-5344/3740}

Contemporaneamente, a Altmetria tem sido temática de interesse nos estudos sobre a comunicação científica. Tais estudos apontam essa abordagem quantitativa como uma forma significativa no mapeamento da produção científica circulante na web social. Neste escopo, esta pesquisa tem como objetivo analisar os indicadores altmétricos dos periódicos da Ciência da Informação com Qualis A1 (Capes), tendo como intuito levantar as variáveis: fontes de informação cujos artigos tiveram maior atenção online; periódicos que mais tiveram artigos com impacto nas redes sociais; periódicos que possuem ferramentas altmétricas incorporadas ao site institucional e; periódicos que possuem perfis em mídia social.Do ponto de vista metodológico, a pesquisa se caracteriza como quantitativa e qualitativa, do tipo exploratória que busca fazer uma 
análise comparativa a partir de indicadores altmétricos extraídos por meio da ferramenta Altmetric Explorer. Os resultados indicam que a maioria das publicações possui menções em fontes on-line. Nota-se que o nível de atenção on-line é maior para os artigos mais recentes. Assim como já observado em outros estudos altmétricos, $o$ Mendeley e o Twitter são as principais redes sociais de circulação de resultados de pesquisas. Destaca-se um número significativo de menções aos artigos em notícias, o que indica que, especificamente na área da Ciência da Informação, os indicadores altmétricos podem contribuir com os estudos relacionados à divulgação científica na área. Observou-se que a maioria das revistas mais mencionadas não é de interesse específico da Ciência da Informação, bem como que grande parte dos periódicos tem perfis em redes sociais. Conclui-se que há um contexto favorável para a utilização de indicadores altmétricos como recurso complementar na avaliação da produção científica, tanto como contribuição nos estudos da comunicação científica.

Palavras-chave: Altmetria. Atenção on-line. Periódicos Científicos. Ciência da Informação.

\section{Alternative metrics of Information Sciencejournals}

Currently, the Altmetrics has been topicof interest in studies on scientific communication that has pointed to this quantitative approach as a significant way for mapping scientific production circulating in the social web. In this scope, this research has as objective to analyze the altmetric indicators of the Information Science journals with Qualis A1 (Capes), aiming at raising the variables: sources of information whose articles had more attention on-line; newspapers that had most articles that impact on social networks; periodicals that have altmetric tools incorporated into the institutional site; newspapers that have social media profiles. From the methodological point of view, it is characterized as a quantitative and 
qualitative research, of the exploratory type that seeks to make a comparative analysis from altmetric indicators extracted through the tool Altmetric.com. The results indicate that most publications have mentions in on-line sources. Note that the level of on-line attention is higher for newer articles. As already seen in other altmetric studies, Mendeley and Twitter are the main social networks for circulating search results. There is a significant number of references to articles in news, which indicates that specifically in the area of Information Science, altmetric indicators may contribute to studies related to scientific dissemination in the area. It wasobserved that most of the mentioned journals are not of interest specific to Information Science, as well as that most of the journals have profiles in social networks. It is concluded that there is a favorable context for the use of altmetric indicators as a complementary resource in the evaluation of scientific production, both as a contribution in studies of scientific communication and in studies on scientific dissemination.

Keywords: Altmetria. On-line attention. Scientific periodicals. Information Science.

Recebido em 01.11.2018 Aceito em 07.12.2020

\section{Introdução}

Os índices de citação que medem o impacto da pesquisa científica derivam-se de metadados formados pelo conjunto de dados que representam a comunicação científica indexada em bases de dados tradicionalmente reconhecidas no âmbito acadêmico internacional como, por exemplo, a Webof Science da Thomson Reuters e a Scopus da Elsevier. Tais índices de citação não espelham o impacto imediato de uma comunicação científica e a maior parte deles abarca especificamente um tipo de publicação - o artigo de periódico, ou seja, os artigos publicados em revistas científicas que não se encontram indexados nas grandes bases de dados não são contabilizados para a construção dos índices de citação.

No contexto das redes sociais, que se caracterizam pela fluidez e rapidez da circulação da informação, surgiram ferramentas capazes de fazer a quantificação instantânea de visualizações e repercussões de 
resultados de pesquisas circulantes nesse ambiente social. A percepção sobre o impacto dessas redes no meio acadêmico aliadaàs tecnologias de monitoramento da informação científica impulsionaramo direcionamento das pesquisas sobre as métricas alternativas que se respaldam nos modelos vigentes de comunicação entre os cientistas.

O aumento da participação efetiva de cientistas nas redes sociais acadêmicas, como o Mendeley e o ResearchGate, e nas redes sociais de abrangência genérica, como o Twitter e o Facebook, contribuiu para maior democratização do conhecimento científico. Conforme Bueno (2010, p. 5), a divulgação científica visa a

[...] democratizar o acesso ao conhecimento científico e estabelecer condições para a chamada alfabetização científica. Contribui, portanto, para incluir os cidadãos no debate sobre temas especializados que podem impactar sua vida e seu trabalho, a exemplo de transgênicos, células tronco, mudanças climáticas, energias renováveis e outros itens.

A possibilidade de monitoramento da atenção on-line da comunicação científica circulante na WebSocial, pautado no acesso amplo que contempla leitores e interagentes que estão fora extrato acadêmico são a base daAltmetria. Trata-se de uma abordagem quantitativa que permite medir e monitorar o alcance e o impacto da produção científica por meio de interações on-line, sendo também conhecida como "métricas alternativas", posto que "alternativa" se refere a medidas tradicionais de sucesso acadêmico, como contagem de citações, prestígio do periódico (Fator de Impacto) e índice $\mathrm{H}$ do autor.

Altmetrics, traduzida como Altmétricas ou ainda Altmetria, refere-se a fontes de dados, ferramentas e métricas que fornecem informações potencialmente relevantes sobre o impacto de resultados de pesquisas, por exemplo: o número de vezes que uma publicação foi tweetada, compartilhada no Facebook ou lida noMendeley. "A Altmetria abre as portas para uma interpretação mais ampla do conceito e formas diversas de análise de impacto" (WALTMAN; COSTAS, 2014, p. 433).

Conforme Fausto (2013, on-line),

a Altmetria é um campo emergente, surgido em 2010, que requer mais investigações sobre sua validade e significado, mas que se apresenta promissor para a compreensão do impacto da pesquisa científica na web social; um campo que abre novas perspectivas para uma abordagem multidimensional sobre a visibilidade e o alcance das pesquisas, considerando inclusive uma nova relação entre ciência e sociedade. 
As fragilidades das métricas tradicionais foram reforçadas com o desenvolvimento das métricas alternativas. $O$ tempo para a obtenção de índices de citação, por exemplo,é um ponto de crítica para a medição do impacto de um artigo científico.ParaNassi-Calò ${ }^{1}$ (2017),os índices bibliométricos baseados em citações são criticados porquea prática de citação de artigos é extremamente complexa e influenciada por inúmeros fatores. Segundo o autor, os verdadeiros motivos para citar um e não outro artigo não traduza qualidade, validade ou relevância dos estudos. O princípio dos indicadores altmétricos, por sua vez, é a medição imediata das menções dos resultados de pesquisa nas redes sociais, avançando no que concerne ao tempo para observação do impacto de uma publicação.

No Brasil, há uma produção crescente sobre a Altmetria como abordagem quantitativa para a avaliação da produção científica(ALPERIN, 2014; ARAÚJO, 2014; ARAÚJO, 2015; NASCIMENTO; ODDONE, 2014). $\mathrm{Na}$ área da Ciência da Informação (CI), Araújo (2015)analisa artigos de periódicos da Ciência da Informação Qualis $A$, presentes em mídias sociais.

A partir da premissa de que os resultados dessas pesquisas, bem como o próprio desenvolvimento da área, podem ter impactado o comportamento dos editores a respeito da inserção dos periódicos no contexto da WebSocial, objetiva-se nesta pesquisaelaborar e analisar indicadores altmétricos de periódicos nacionais e internacionais da Ciência da Informação avaliados como Qualis A1 pelo Sistema Brasileiro de Avaliação de Periódicos, mantido pela CAPES (Coordenação de Aperfeiçoamento de Pessoal de Nível Superior), tendo como intuito refletir sobre a aderência dos periódicos dessa área no contexto das métricas alternativas.Espera-se com este estudode abordagem altmétricaapontar um panorama dos periódicos da CI no que se refere à aderência àWebSocial como forma de medição do impacto de suas publicações.

\section{Medidas altmétricas de impacto para avaliação da produção científica}

Os estudos sobre os indicadores altmétricos, baseados nas métricas da circulação da informação científica em redes sociais, trazem uma série de considerações a respeito de sua utilização para a medição da produção científica.

\footnotetext{
${ }^{1} V$ isto que a literatura científica que fundamentou a pesquisa é majoritariamente publicada em língua inglesa, ressalta-se que todas as citações da literatura estrangeira foram traduzidas pelas autoras.
} 
Alguns estudos buscam um entendimento sobre o correlacionamento entre as métricas alternativas e tradicionais. Thelwall et al. (2013) realizaram um estudo comparativo entre 11 indicadores altmétricos e indicadores de citações da Web of Science e PubMed. Os autores,por meio de coeficientes de correlação, verificaram que 0 grau de correlacionamento dos indicadores altmétricos e dos índices de citação parece ser baixo e depende diretamente dos fatores tempo de publicação e fonte da atenção. Os autores alertam que além dos escoresaltmétricos, o tempo de publicação deve ser considerado na classificação de resultados de pesquisa.

Torres-Salinas, Cabezas-Clavijo e Jimenez-Contreras (2013) também compararam indicadores tradicionais, como a citação, com indicadores altmétricos de uma amostra da produção científica espanhola na área de Comunicação. Os autores verificaram que os trabalhos mais citados são os que obtiveram maior impacto altmétrico. Embora a pesquisa tenha apontado as deficiências que essas métricas apresentam, indicaram o papel que elas podem desempenhar para medir o impacto da pesquisa por meio de plataformas 2.0.

Já Haustein, Costas e Lariviere (2015) analisaram 1,3 milhão de artigos indexados Webof Science (WoS), publicados em 2012. Os autores fizeram uma análise comparativa entre as citações nessa base e contagens desses artigos em mídias sociais. Os resultados revelaram que tanto as citações quanto as métricas de mídia social aumentam com a extensão da colaboração e com o número de autores citados nos trabalhos. Outros achados revelaram-seda mesma forma importantes. Os editoriais e as notícias, por exemplo, raramente são citados, mas esses tipos de documentos são os mais populares no Twitter; os artigos mais extensos atraem mais citações e são menos mencionados em mídias sociais; os artigos da Ciências Sociais e Humanas são mais frequentemente encontrados nas plataformas de mídias sociais, resultado oposto do que se observa para as citações nessa área. Os autores sugerem que os fatores que impulsionam as menções em mídias sociais e as citações são diferentes. Portanto, as métricas de mídias sociais podem funcionar como complementos e não como alternativas para outros tipos de indicadores.

Conforme Melero (2015), as métricas bibliométricas baseadas em citações e contagem de publicações e as métricas alternativas podem ser empregadas em conjunto de maneira a proporcionarem uma visão sobre o impacto imediato ou a longo prazo de resultados de pesquisa. Dessa forma, tornam-se na verdade complementaresà tradicional e não alternativas, ou seja, aumentam as variáveis de observação sobre a repercussão de resultados acadêmicos nesse âmbito e sobre a repercussão precoce do impacto social. 
Outros estudos tiveram como foco levantar e analisar as fontes de informação utilizadas para a obtenção de indicadores altmétricos, dentre os quais se destacam os de Robinson-Garcia et al. (2017), Schmitt e Jäschke (2017), Ke, Ahn e Sugimoto (2017), Ravenscroft et al. (2017) e Asnafi (2017). Essas pesquisas utilizaram as fontes de informação ReseachGate, Mendeley, CrossREF e Twitter para a obtenção de indicadores altmétricos. Dentre essas fontes, o Twitter tem maior destaque entre os estudos.

O Twitter é uma rede social dinâmica e de grande alcance. Por possuir limites de caracteres para a divulgação de conteúdo, consegue fazer uma representação simplificada de um trabalho. A grande ressalva quanto à utilização do Twitter para a construção de indicadores altmétricos são os bots (diminutivo de robot), ou seja, os softwares que controlam contas no Twitter. Com a utilização de aplicativos épossível realizar postagens anônimas que podem atrapalhar a análise da menção (impacto), isso porque essas postagens são automáticas e portantoindissociadas das postagens geradas diretamente por humanos (ROBINSON-GARCIA et al.,2017).

Em relação às ferramentas para construção de indicadores altmétricos, a literatura aponta o Altmetric como a mais utilizada (GASPARYAN et al., 2017; ROBINSON-GARCIA, et al., 2017; RAVENSCROFT et al., 2017; ARAÚjO et al., 2017). Essa ferramenta refere-se a um serviço on-line especializado em coletar e apresentar dados altmétricos das principais fontes de menções de pesquisas científicas. Além de ser uma ferramenta, é considerada também uma fonte de pesquisa pela disponibilidade de captura de inúmeros indicadores altmétricos. Essa ferramenta utiliza vários métodos para coleta das menções de pesquisas científicas, como reconhecimento de hyperlink que direciona ao artigo original ou mecanismos de rastreio de notícias que buscam menções a artigos, revistas e autores.

\section{Percurso Metodológico}

Esta pesquisa pode ser considerada bibliográficapor discorrer sobre a temáticaAltmetria expressa na literatura. Trata-se ainda de uma pesquisa do tipo exploratória e descritiva, a qual foram utilizadas as fontes de dados Qualis-Periódicos para a seleção do corpus de periódicos da Ciência da Informação, a Ferramenta Altmetrics Explorer para o levantamento do Score Altmétricoe indicadores das menções on-line dos artigos publicados nos periódicos selecionados para essa pesquisa e o próprio site desses periódicos para levantar os plug-ins de ferramentas altmétricas incorporadas às suas homepages. A natureza da pesquisa é quantitativa e qualitativa, buscando a manipulação e compreensão dos 
indicadores levantados. Têm-se então como objeto desta pesquisa os periódicos da Ciência da Informação.

A seguir apresentam-se os procedimentos de coleta de dados, mesclando-se com a caracterização das fontes e ferramentas que foram utilizadas.

O Qualis-Periódicos é um sistema usado para classificar a produção científica dos programas de pós-graduação no que se refere aos artigos publicados em periódicos científicos. Tal processo foi concebido para atender as necessidades específicas do Sistema Nacional de PósGraduação (SNPG) e é baseado nas informações fornecidas pelos programas de pós-graduação nacionais, por meio do aplicativo Coleta de Dados, na Plataforma Sucupira. Como resultado, disponibiliza uma lista com a classificação dos veículos utilizados pelos programas de pósgraduação para a divulgação da sua produção.

$\mathrm{Na}$ Plataforma Sucupira, selecionaram-se as classificações de periódicos do quadriênio 2013-2016 e a área de avaliação Comunicação e Informação em Classificação A1, totalizando assim uma listagem com 47 periódicos.

$\mathrm{Na}$ ferramenta Altmetric Explorer, ferramenta da família Altmetric, pesquisou-se em 'journalorcollection' e pelo ISSN correspondente a cada periódico, conforme Figura 1.

Figura 1 - Busca por periódicos na ferramenta Altmetric Explorer

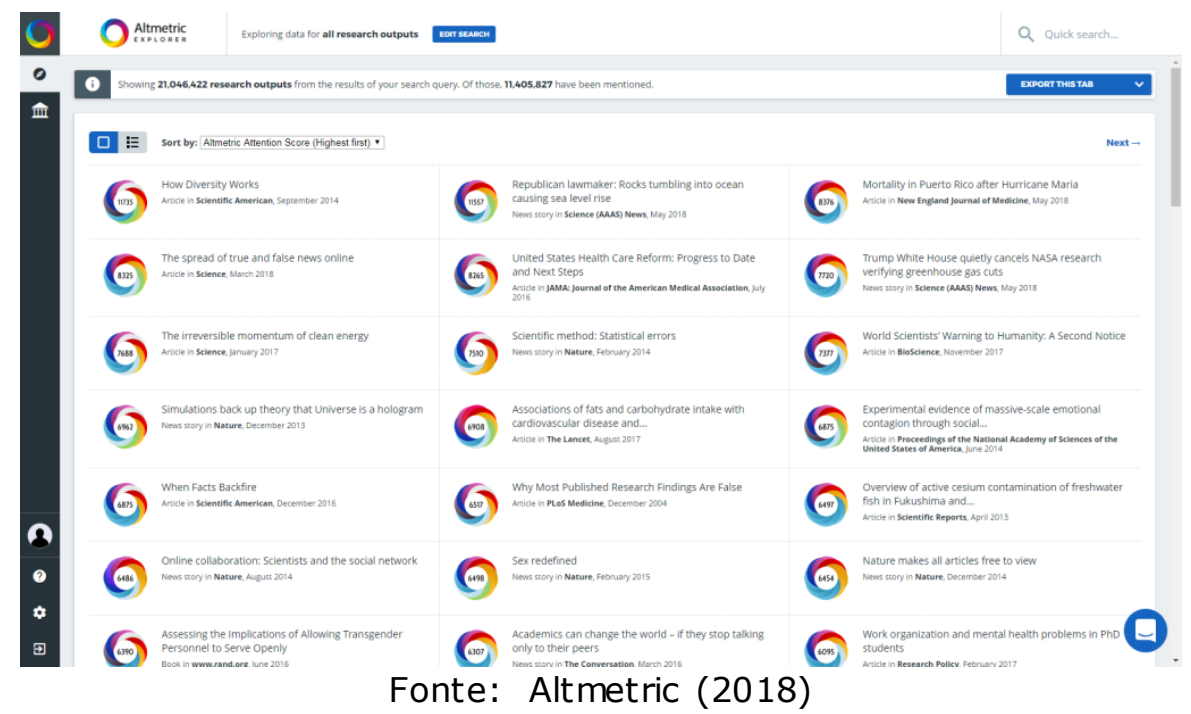

Após o levantamento dos indicadores altmétricos na ferramenta Altmetric Explorer, os dados foram exportados para o software Microsoft Excel (em formato CSV) e depois exportado para a ferramenta Talend Open Studio. Esta ferramenta é open source paraExtractTransformLoad ${ }^{2}$

\footnotetext{
${ }^{2}$ ETL, do inglês ExtractTransformLoad (Extração Transformação Carregamento), são ferramentas de software cuja função é a extração de dados de diversos sistemas.
} 
(ETL) e tem como função permitir a integração de dados facilitando a análise. Na Figura 2 apresenta-se essa ferramenta.

Figura 2 - Talend Open Studio 3

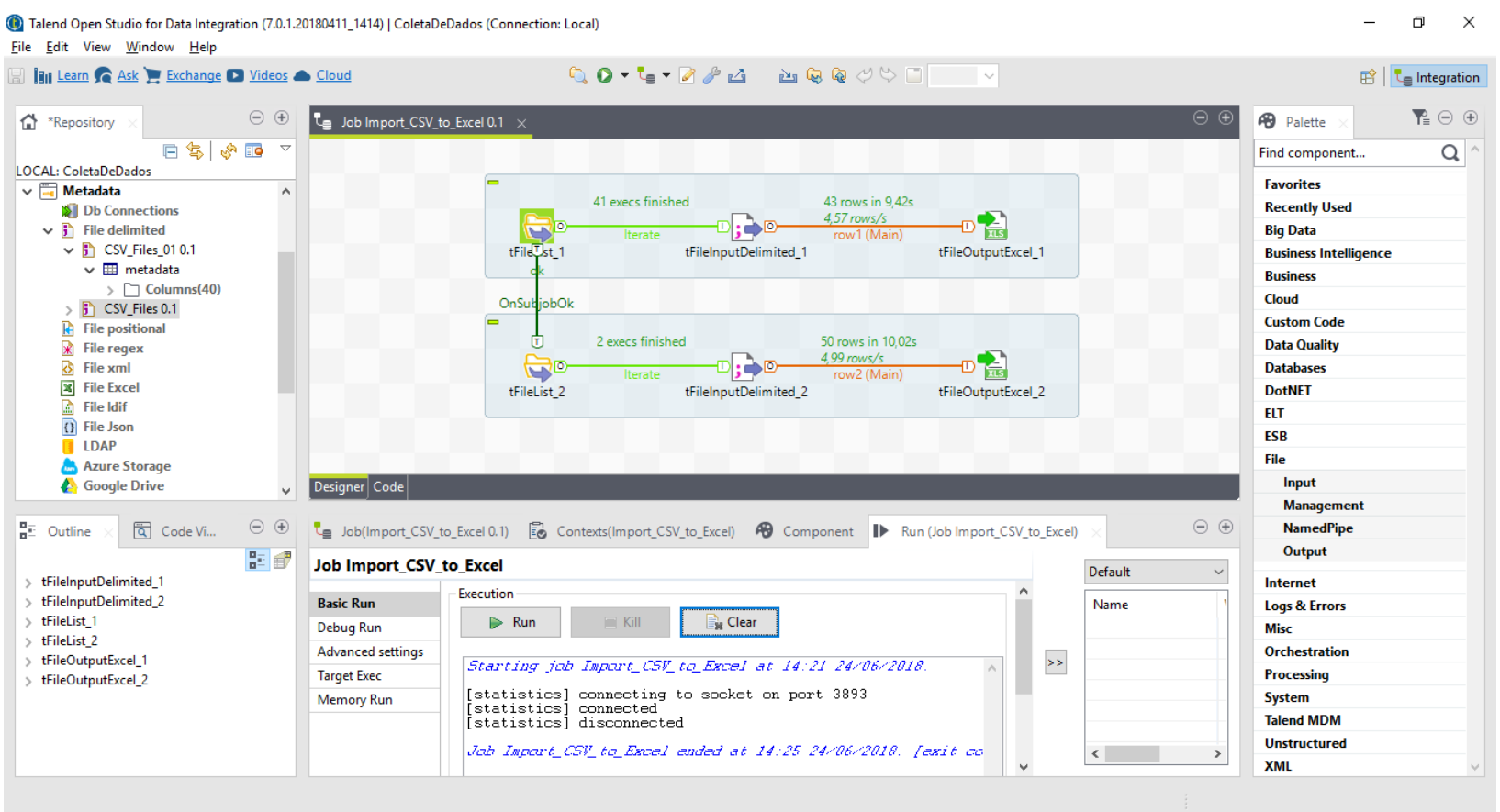

Fonte: Elaboração das autoras no momento da extração dos dados

Todos os arquivos estavam separados e a ferramenta Talent foi utilizada para agrupar e padronizar todos os dados em uma única base de dados no Microsoft Excel. Após a padronização, procedeu-se à elaboração de gráficos e tabelas com os indicadores sobre: a relação entre o ano de publicação dos trabalhos e a quantidade de menções on-line; as principais fontes das menções on-line desses artigos; e os principais periódicos de acordo com a quantidade de menções on-line. Paralelamente, pesquisouse nos sites de cada periódico se possuíam perfil em alguma rede social e se havia ferramentas de mensuração da atenção on-line incorporadas àhomepage. Além disso, buscou-se verificar quais os pesos atribuídos pelo algoritmo da ferramenta Altmetric Explorer para apresentar os indicadores de atenção on-line de cada artigo.

Os dados levantados foram analisados com base nas seguintesvariáveis: relação entre o ano de publicação e a quantidade de menções on-line; principais fontes das menções on-line dos artigos; principais periódicos de acordo com a quantidade de menções on-line; escores altmetricos dos artigos com maior representatividade; leitores dos artigos com maior score de acordo com seu status profissional e por

\footnotetext{
${ }^{3}$ www.talendforge.org
} 
disciplina; periódicos que possuem plug-ins de ferramentas altmétricas e; periódicos científicos que possuem perfil em alguma rede social. Essas variáveis foram discutidas com respaldo na literatura, buscando destacar os indicadores a partir da análise das métricas alternativas dos principais periódicos nacionais e internacionais da Ciência da Informação.

\section{Análise das métricas alternativas dos periódicos nacionais e internacionais da Ciência da Informação}

Tradicionalmente, o impacto científico é medido por meio de indicadores bibliométricos, como os índices de Fator de Impacto da Web of Knowledge (Thomson Reuters) e o Scimago Journal Rank (Elsevier), cujo foco de análise são as publicações indexadas nessas plataformas, por meio das quais se mensura o número de citações que um periódico tem num período de dois anos dividido pelo número de artigos publicados. Por outro lado, o Índice Hanalisa o impacto da produção científica individual dos cientistas ou dos periódicos. No índice $\mathrm{H}$, relaciona-se o número de publicações com o número de citações e cria-se um número que somente tenderá a subir se a qualidade das publicações, por conseguinte aumentar. Essas métricas bibliométricas têm sustentado discussões sobre suas limitações, pois questiona-se se elas realmente refletem o mérito da produção científica e dos pesquisadores.

Embora sejam medidas tradicionais, as citações não podem ser usadas para medir o impacto da pesquisa em outros estratos da sociedade,razão pela qual Bornmann (2014) afirma que a Cienciometria busca novas possibilidades para mensuração da produção científica, principalmente por meio das avaliações de impacto social e os efeitos dos resultados das pesquisas na sociedade. Nessa perspectiva, entende-se a Altmetria como uma possibilidade atraente para medir o impacto social (MOHAMMADI; THELWALL, 2014).

Desse modo, apresentam-se nesta pesquisa dados altmétricos de periódicos da Ciência da Informação referentes à quantidade de menções on-line dessas publicações, a relação entre o ano de publicação e a quantidade de menções on-line e as principais fontes das referidas menções. Além desses dados altmétricos, verificou-se se os periódicos possuem ferramentas de mensuração altmétrica incorporadas à sua homepage e se possuem contas em redes sociais.

$\mathrm{Na}$ ferramenta Altmetric Explorer, pesquisaram-se individualmente as 47 revistas classificadas como Qualis A1 na área de Informação e Comunicação.O resultado desse levantamento demonstrou que a maioria dessas publicações (89\%) possuimenções em fontes on-line. Somente cinco periódicos $(11 \%)$ não possuem menção dessa natureza.

Nas 42 revistas que possuem menções on-line, foram identificados 1.881 artigos que totalizaram 128.751 menções em fontes on-line. Ao se 
observar o ano de publicação em relação à quantidade de menções on-line (Gráfico1), nota-se que o nível de atenção on-line aumenta a partir de 2007, com destaque para os anos $2016(n=16.667) ; 2013(n=16.426)$; $2015(n=12.623) ; 2017 \quad(n=11.894) ; 2012 \quad(n=10.605)$ e 2007 $(n=10.056)$.

$$
\begin{gathered}
\text { Gráfico } 1 \text { - Relação entre o ano de publicação e a quantidade de menções on- } \\
\text { line }
\end{gathered}
$$

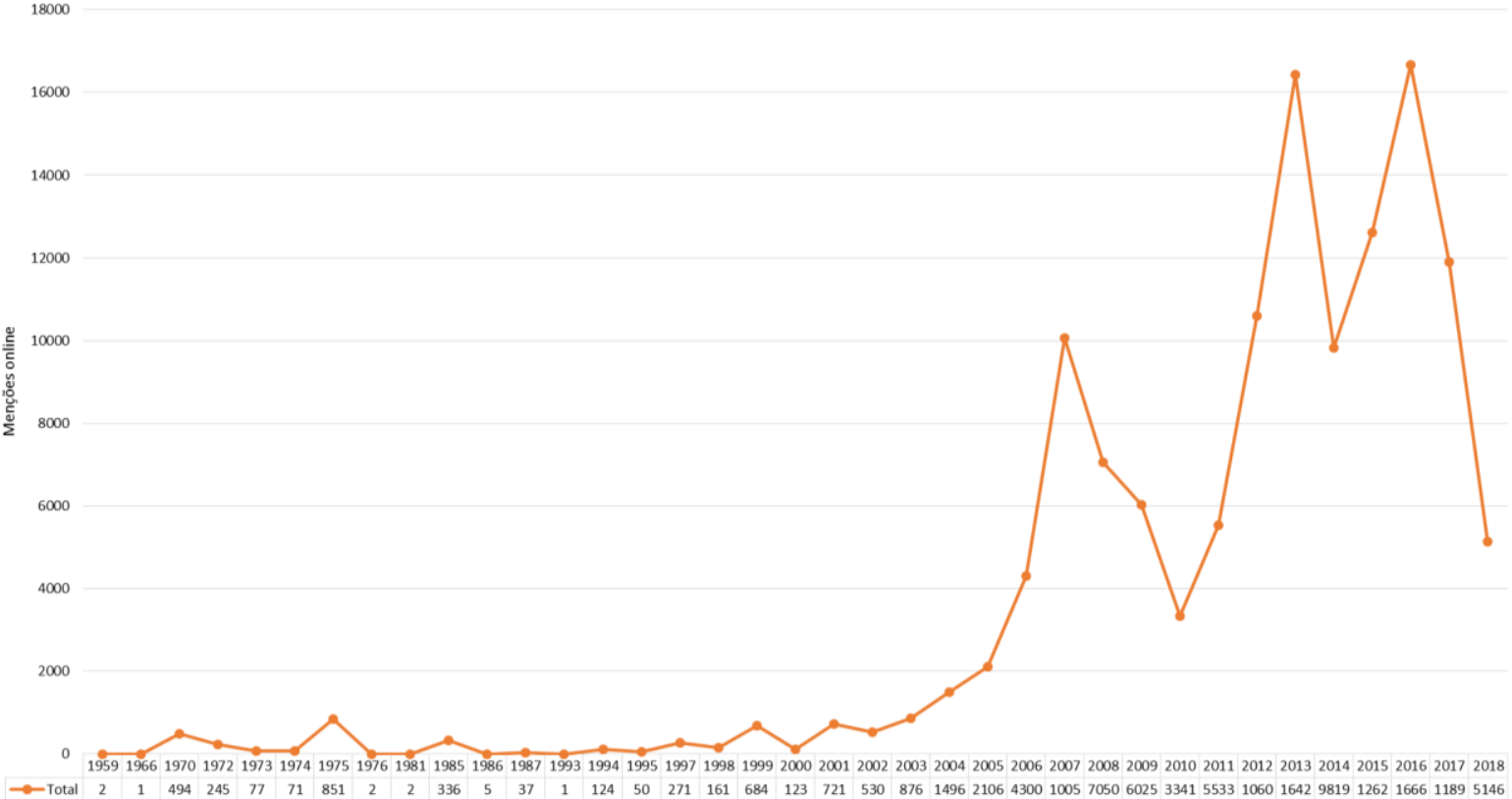

Fonte: Elaboração das autoras

No Gráfico 1, observa-se que os artigos mais recentes são mais mencionados nas redes sociais, o que denota a importância da Altmetria para o monitoramento instantâneo de um resultado de pesquisa. Embora o ano tenha se destacado como fator que influenciou a maior atenção online, Holmberg e Vainio (2018) verificaram que o nível de atenção on-line recebida é uma soma de muitos fatores, inclusive existem diferenças específicas entre as plataformas onde a atenção foi recebida. Os autores ainda ressaltaram que é importante analisar todos esses fatores para compreender se a atenção on-line reflete o impacto social ou científico.

Vale ressaltar que a palavra altmetrics foi mencionada pela primeira vez em um tweet de Jason Priem em 2010 e, posteriormente, foi proposto no Manifesto Altmétrico que destaca que a Altmetria expande a visão do que é impacto e do que está causando-o. Adie e Roe (2013) ressaltam que a altmetria apresenta uma alternativa à prática atual de mensurar apenas com contagens de citações e fatores de impacto de periódicos,evoluindo para a análise quantitativa do impacto por meio de novas abordagens. Assim, as medições altmétricas não substituirão a 
avaliação baseada em citações, porém complementarão as análises demonstrando outros aspectos do impacto que uma pesquisa teve.

Desse modo, com as novas tecnologias de informação e comunicação, os acadêmicos utilizam cada vez mais a web para realizar suas pesquisas, pois a facilidade do compartilhamento em nuvem favorece a elaboração de pesquisas em parcerias, assim como os gerenciadores onlinede referências bibliográficas como EndNoteWeb, Zotero e Mendeley facilitam o compartilhamento de fontes bibliográficas.

No Gráfico 2 evidencia-se, assim como já observado em outros estudos altmétricos (HOLMBERG; VAINIO, 2018), que o Mendeley e o Twitter são as principais redes sociais de circulação de resultados de pesquisas. Essas fontes possuem naturezas diferentes: enquanto o Mendeley é uma rede social acadêmica, na qual participam membros de comunidade acadêmicas, o Twitter é de abrangência genérica.

\section{Gráfico 2 - Principais fontes das menções on- line}

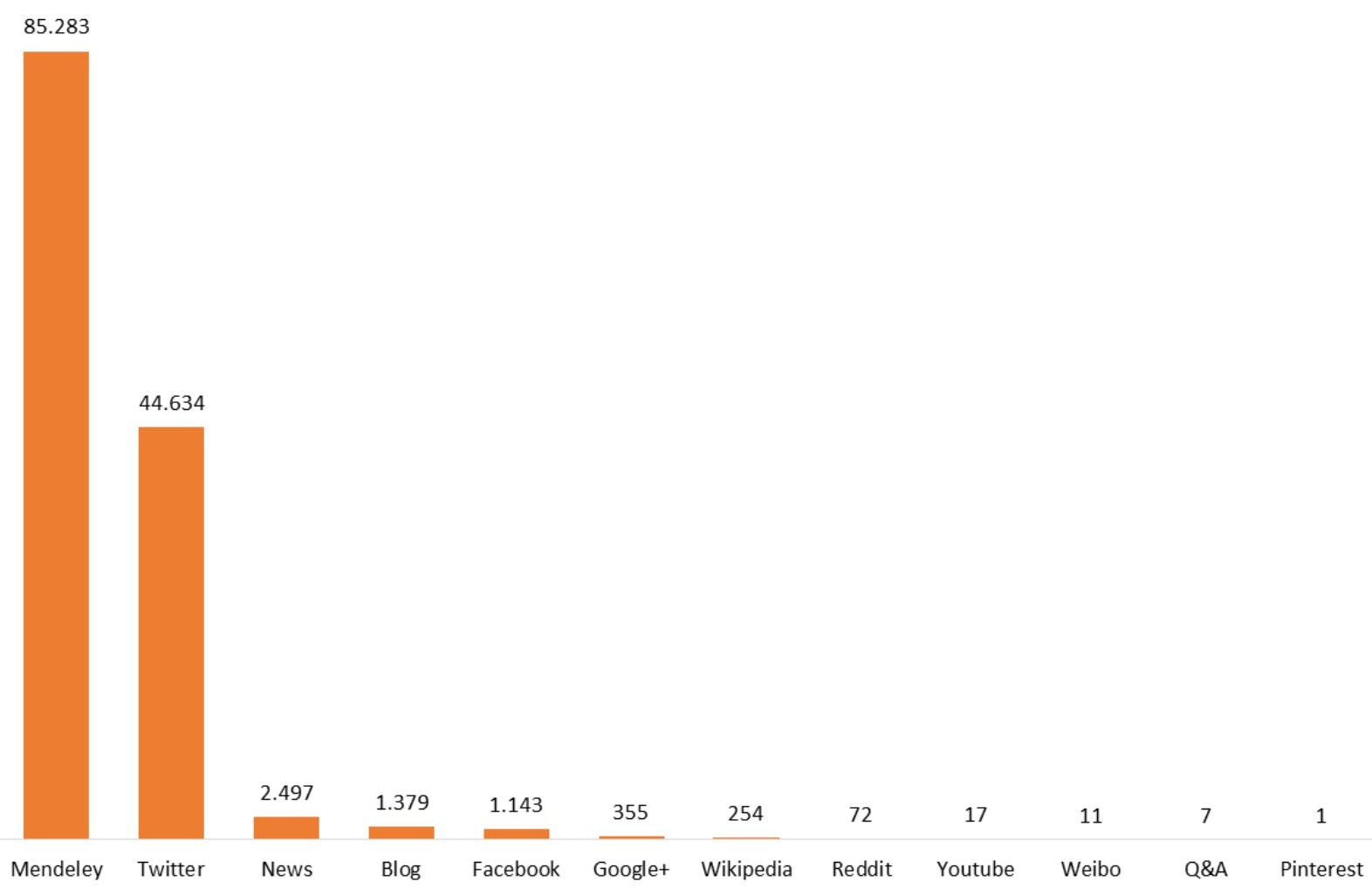

Fonte: Elaboração das autoras

É interessante notar (Gráfico 2) o número significativo de menções aos artigos em notícias. Nesse sentido, destaca-se a importância da "Divulgação científica" considerada por Massola, Crochik e Svartman (2015) como uma expressão "que designa atualmente a transmissão de 
conhecimento científico para um público leigo no assunto". Segundo Luiz (2006, p. 53), divulgar as pesquisas em forma de notícias é uma necessidade dos pesquisadores de "prestar contas à sociedade sobre as realizações na área". Especificamente na área da Ciência da Informação, pode-se notar que os indicadores altmétricos podem contribuir com os estudos relacionados à divulgação científica na área.

No que se refere aos principais periódicos, de acordo com a quantidade de menções on-line, ressalta-se na listagem apresentada na Tabela 1 apenas os que apresentam no mínimo $2 \%$ sobre o total dos escores de menções. Esses representam 88\% das menções aferidas nesta pesquisa.

Tabela 1 - Principais periódicos de acordo com a quantidade de menções online

\begin{tabular}{|c|c|c|c|}
\hline Periódicos & Menções & \% sobre total & $\%$ acumulado \\
\hline Journal of Computer-Mediated Communication & 8.063 & $14 \%$ & $14 \%$ \\
\hline Scientometrics & 5.184 & $9 \%$ & $24 \%$ \\
\hline SAGE Open & 4.756 & $9 \%$ & $32 \%$ \\
\hline Information, Communication \&amp; Society & 4.750 & $9 \%$ & $41 \%$ \\
\hline British Journal of Political Science & 3.345 & $6 \%$ & $47 \%$ \\
\hline Journalism & 2.758 & $5 \%$ & $52 \%$ \\
\hline Digital Journalism & 2.280 & $4 \%$ & $56 \%$ \\
\hline Journalism Studies & 2.152 & $4 \%$ & $60 \%$ \\
\hline Journal of the American Society for Information Science \& Technology & 2.141 & $4 \%$ & $64 \%$ \\
\hline Political Studies & 1.805 & $3 \%$ & $67 \%$ \\
\hline Media, Culture \& Society & 1.570 & $3 \%$ & $70 \%$ \\
\hline Science \& Public Policy (SPP) & 1.532 & $3 \%$ & $72 \%$ \\
\hline Journal of Consumer Culture & 1.404 & $3 \%$ & $75 \%$ \\
\hline Critical Studies in Media Communication & 1.262 & $2 \%$ & $77 \%$ \\
\hline Television \& New Media & 1.177 & $2 \%$ & $79 \%$ \\
\hline Comunicar & 1.149 & $2 \%$ & $81 \%$ \\
\hline Journal of Documentation & 979 & $2 \%$ & $83 \%$ \\
\hline Journal of Librarianship \& Information Science & 947 & $2 \%$ & $85 \%$ \\
\hline Journal of the American Society for Information Science and Technology & 915 & $2 \%$ & $87 \%$ \\
\hline El Profesional de la Información & 843 & $2 \%$ & $88 \%$ \\
\hline
\end{tabular}

Fonte: Elaboração das autoras

Nota-se na Tabela 1 que a maioria das revistas mais mencionadas não é de interesse específico da Ciência da Informação. Nesse conjunto de publicações, as mais direcionadas para essa área são: Scientometrics, JournalofDocumentation, JournalofLibrarianship\&Information Science e El Profissional da Information. Entre as revistas brasileiras com maior número de menções, porém não foram destacadas na Tabela 1, estão: Dados - Revista de Ciências Sociais $(n=229)$, Perspectivas em Ciência da Informação $(n=112)$ e Transinformação $(n=93)$. No trabalho de Araújo (2015) as revistas Perspectivas em Ciência da Informação e Transinformação também se destacaram como as que obtiveram mais métricas alternativas.No entanto, quantitativamente, essas revistas que 
são importantes no contexto brasileiro, ainda possuem um número pequeno de menções em relação às revistas internacionais.

No site $e^{4}$ da ferramenta Altmetric consta a explicação mais apurada acerca do cálculo da pontuação de atenção altmétrica. A pontuação é derivada de um algoritmo automatizado e representa uma contagem ponderada da quantidade de atenção que captam nas diferentes fontes on-line, atribuem-se diferentes pesos. $\mathrm{Na}$ Tabela 2,apresentam-se osescores de atenção dos artigos em relação às fontes de coleta atribuídos pela ferramenta Altmetric Explorer.

Tabela 2 - Pesos atribuídos pelo algoritmo do Altmetric Explorer

\begin{tabular}{l|c}
\hline \multicolumn{1}{c|}{ Fontes } & Peso \\
\hline Notícia & 8 \\
\hline Blogs & 5 \\
\hline Twitter & 1 \\
\hline Facebook & 0,25 \\
\hline Sina Weibo & 1 \\
\hline Wikipedia & 3 \\
\hline Policy Documents (por fonte) & 3 \\
\hline Q \& A & 0,25 \\
\hline F1000/ Publons / Pubpeer & 1 \\
\hline Youtube & 0,25 \\
\hline Reddit / Pinterest & 0,25 \\
\hline LinkedIn & 0,5 \\
\hline Open Syllabus & 1 \\
\hline Google+ & 1 \\
\hline
\end{tabular}

Fonte: Atmetric (2018)

O Attention Score é útil para classificar os resultados da pesquisa com base na atenção. Embora não se possa relacionar esse índice com a qualidade do artigo em si, é possível ler e ponderar as discussões vinculadas a cada artigo. Essa pontuação do escoreé baseada nos tipos de atenção que a Altmetric monitora - especificamente links ou salvamentos de artigos acadêmicos, livros e conjuntos de dados. Como possíveis limitações a esse escore, ressaltam-se que áreas de assuntos diferentes geralmente não são diretamente comparáveis, ou seja, um artigo de física "popular" pode ter uma pontuação de atenção altmétrica muito menor do que um artigo da área de genética, por exemplo. Em alguns casos raros, a Pontuação de Atenção Altmétrica pode flutuar ao longo do tempo. Flutuações podem acontecer por vários motivos, como quando os tweets são removidos pelo tweeter original ou se as contas do Twitter são

\footnotetext{
${ }^{4}$ https://help.altmetric.com/support/solutions/artides/6000060969-how-is-the-altmetric-scorecalculated-
} 
consideradas "tendenciosas" de acordo com os algoritmos automatizados da ferramenta. Além disso, ocasionalmente são feitas alterações no algoritmo, a fim de garantir que a pontuação seja um reflexo preciso do alcance e da legitimidade da atenção atribuída a uma saída de pesquisa.

Assim, destaca-se nesteartigo a publicação de Moira Burke e Robert E. Kraut intitulada "The Relationship between Facebook Use and WellBeing depends on Communication Type and Tie Strength", publicado no Journal of Computer-Mediated Communicationem 2016, com escore $n=1.048$. O referido artigo teve menções em notícias $(n=127)$, em blogs $(n=12)$, Twitter $(n=112)$, Facebook $(n=2)$, Mendeley $(n=173)$ e no CiteULike $(n=1)$.

Grande parte dessas menções foi capturada no Mendeley $(n=112)$ e, no que concerne à origem das menções nessa rede social, verificou-se que $68 \%$ foram realizadas por membros do público em geral $(n=76), 22 \%$ de cientistas $(n=25)$ e $10 \%$ de Comunicadores de ciência (jornalistas, blogueiros, editores) $(n=11)$. O Altmetric categoriza os usuários do Twitter com base no histórico de postagem e nas informações de perfil. Quando os dados do Twitter estão disponíveis para um artigo, as contagens de cada categoria de usuário e os dados de geolocalização são incluídos na guia Informações demográficas da página de detalhes do artigo. Para compilar informações sobre o perfil dos membros que twittarama publicação, a ferramenta analisou as palavras-chave nas descrições de perfil, os tipos de periódicos aos quais os usuários se conectou e as listas de seguidores para atribuir a cada perfil uma categoria. Assim, consideram:

- Membro do público - alguém que não tem links para literatura acadêmica e não se encaixa em nenhuma das categorias abaixo;

- Pesquisador - alguém familiarizado com a literatura;

- Praticante - um clínico ou pesquisador que está trabalhando na ciência ou tecnologia;

- Comunicador de ciência - alguém que se conecta

frequentemente a artigos científicos de diversos periódicos / editores. (Fonte: Altmetrics Explorer).

No que se refere aos dados compilados a partir de estatísticas de usuários Mendeley, constatou-se que 171 leitores pelo menos salvaram os metadados dos artigos nesse gerenciador pessoal de referências bibliográficas. Na Tabela 3,apresentam-se os leitores de acordo com seu status profissional e por meio dela pode-se observar que a maioria dos usuários do Mendeley que utilizou esse artigo possui título de doutor, perfazendo $32 \%(n=54)$. 
Tabela 3 - Leitores de acordo com seu status profissional - Mendeley

\begin{tabular}{l|c|c}
\hline Leitores por status profissional & Qtd. & \% s/Total \\
\hline Doutor & 54 & $32 \%$ \\
\hline Bacharel & 22 & $13 \%$ \\
\hline Mestre & 20 & $12 \%$ \\
\hline Pesquisador & 19 & $11 \%$ \\
\hline Doutorando & 10 & $6 \%$ \\
\hline Outros & 46 & $27 \%$ \\
\hline
\end{tabular}

Fonte: Altmetric Explorer (2018)

No que se refere ao perfil dos usuários do referido artigo por disciplinas (Tabela 4), verificou-se que as áreas de Ciências Sociais representam 28\%; Psicologia 26\%; e Ciência da Computação 15\%.

Tabela 4 - Leitores por disciplina - Mendeley

\begin{tabular}{l|c|c}
\hline Leitores por disciplina & Qtd. & \% s/Total \\
\hline Ciências Sociais & 48 & $28 \%$ \\
\hline Psicologia & 45 & $26 \%$ \\
\hline Ciência da Computação & 25 & $15 \%$ \\
\hline Não especificado & 18 & $11 \%$ \\
\hline Negócios, Gestão e Contabilidade & 13 & $8 \%$ \\
\hline Outras & 22 & $13 \%$ \\
\hline
\end{tabular}

Fonte: Altmetric (2018)

Destacam-se também outros artigos com maioresescoresaltmétricos: "The decline in the concentration of citations, 1900-2007" ( $n=928)$; "Objectivity and realms of explanation in academic journal artides concerning sex/gender: a comparison of Gender studies and the other social sciences" $(n=928)$; "The Extended iSelf: The Impact of iPhone Separation on Cognition, Emotion, and Physiology" $(n=812)$; "Food Art Does Not Reflect Reality: A Quantitative Content Analysis of

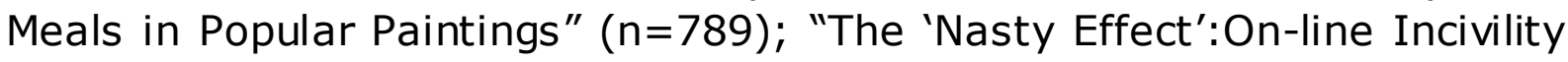
and Risk Perceptions of Emerging Technologies" $(n=693)$; "The Benefits of Facebook 'Friends': Social Capital and College Students' Use of On-line Social Network Sites" $(n=497) ;$ "Declines in American Adults' Religious Participation and Beliefs, 1972-2014" $(n=484)$; "Mapping the antivaccination movement on Facebook" $(n=449)$; e "Journalism, not

\footnotetext{
${ }^{5}$ AltmetricDetails Page. Disponível em: https://www.altmetric.com/explorer/outputs?show_details=10056151\&title=The\%20Relationship\% 20between\%20Facebook\%20Use\%20and\%20Well-

Being\%20depends\%20on\%20Communication\%20Type\%20and\%20Tie\%20Strength\%20
} 
'cheerleading': An ombudsman's paradigm repair in the JNU sedition case in India" $(n=389)$.

Dentre os artigos de revistas brasileiras, destacam-se: "Redistributionanddevelopment? The politicaleconomyofthe bolsa família program" ( $n=30)$ publicado na revista Dados em 2009; "Marketing científico digital e métricas alternativas para periódicos: da visibilidade ao engajamento" ( $n=13$ ) publicado em 2015 na revista Perspectivas em Ciência da Informação; e "A ciência nas regiões brasileiras: evolução da produção e das redes de colaboração científica" $(n=12)$, publicado em 2016 na revista Transinformação.

A aderência dos periódicos aos indicadores altmétricos revelam se esses índices fazem parte das políticas científicas dessas publicações.

Ao se analisar se os periódicos possuem plug-ins de ferramentas altmétricasincorporadas às suas homepages, tais como Altmetric, PlumAnalytics ou ImpactStory, verificou-se que, embora $78 \%(n=35)$ dos periódicos não apresente os indicadores de impacto nas redes sociais de seus artigos científicos de forma instantânea,22\% ( $n=10)$ deles possuem essas ferramentas incorporadas, permitindoassim ao usuário que analisem os indicadores altmétricos de cada artigo. Entende-se, portanto, que seja uma tendência a aderência a esses indicadores como forma complementar aos índices de citação, tendo em vista que essas ferramentasaltmétricas analisam o impacto de um documento por meio de suas menções em redes sociais.

Com esse mesmo direcionamento, realizou-se o levantamentodos periódicos que possuem conta nas principais redes sociais, pois de acordo com Souza e outros (2015) a utilização das redes sociais por periódicos científicos seria uma maneira eficaz de se aproximar do público-alvo e buscar novos usuários, uma vez que, a cada dia, intensifica-se na sociedade global o uso das redes sociais.

Neste artigo, as informações foram extraídas das homepages desses periódicos, nas quais verificou-se se havia indicação dessas redes sociais. Assim, o Gráfico 3 trata da quantidade de periódicos científicos que possuem perfil em alguma rede social. 
Gráfico 3 - Periódicos científicos que possuem perfil em alguma rede social

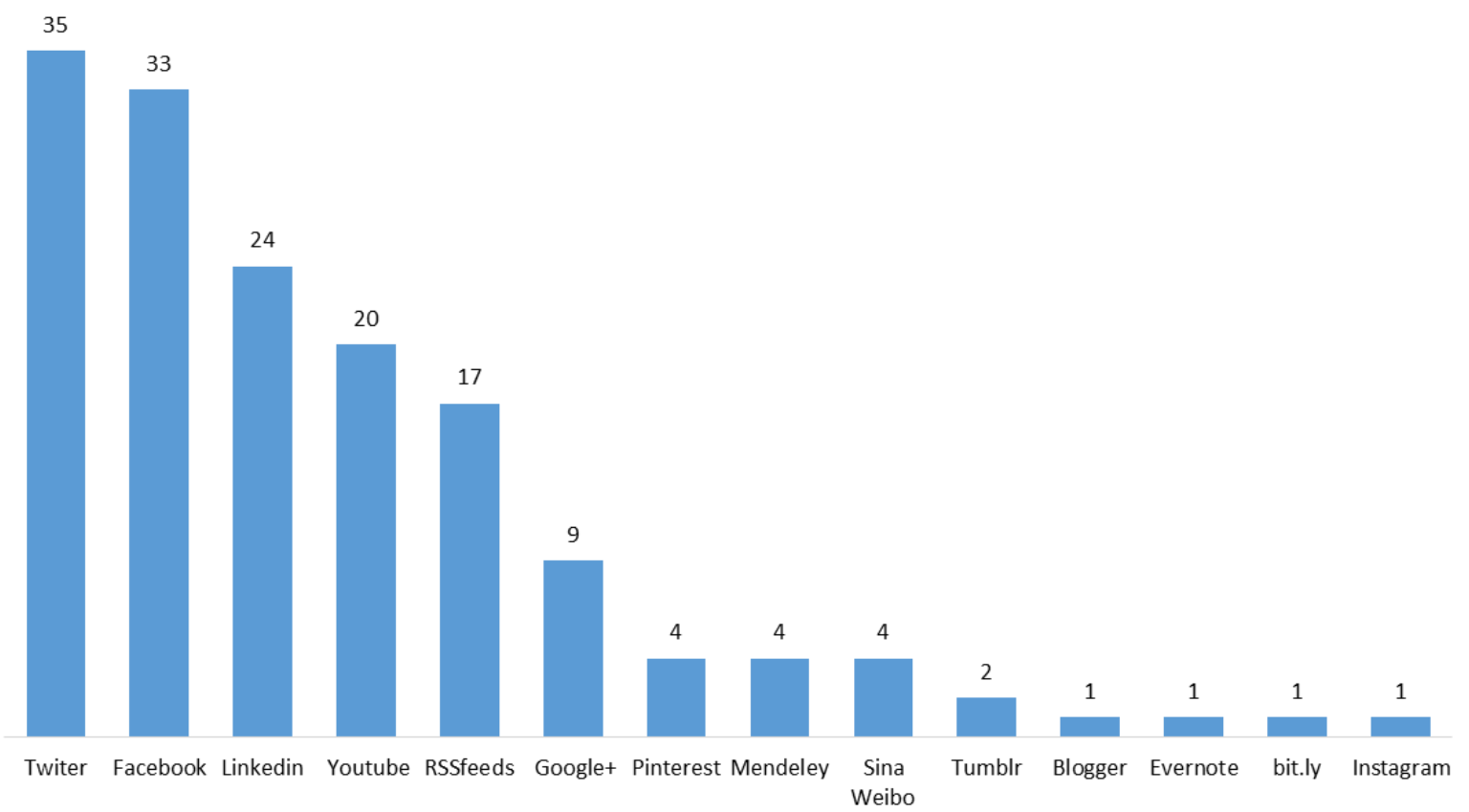

Fonte: Elaboração das autoras

Observou-se que 35 periódicos, ou seja, 83,3\% possuem perfil no Twitter, $78,6 \%$ também possuem perfil no Facebook $(n=33)$ e $57,1 \%$ dos periódicos fazem parte do Linkedin $(n=24)$. Constam ainda no Youtube $(47,6 \%)$, RSSfeeds $(40,5 \%)$, Google+ $(21,4 \%)$ e em outras redes sociais como Pinterest, Mendeley, Sina Weibo, Tumblr, Blogger, Evernote, bit.ly e Instagram. Os dois principais periódicos com mais perfis são o JournalofDocumentation, publicado pela Emerald, que possui perfil em 11 redes sociais, e o JournalofScholarlyPublishing, que apresenta perfil em oitodelas.

Observa-se que tanto as informações sobre a incorporação de ferramentas altmétricas como as apresentadas no Gráfico 3,sobre a aderência a redes sociais, têm influência direta naeditora ao qual pertence o periódico, pois as editoras com maiores recursos buscam destaque tanto nos indicadores tradicionais como nos alternativos.

\section{Considerações finais}

Retomando o objetivo desta pesquisa de elaborar e analisar indicadores altmétricos de periódicos nacionais e internacionais da Ciência da Informação avaliados como Qualis A1 pelo Sistema Brasileiro de Avaliação de Periódicos, mantido pela CAPES (Coordenação de Aperfeiçoamento de Pessoal de Nível Superior), tendo como intuito refletir sobre a aderência dos periódicos dessa área no contexto das métricas 
alternativas, pôde-se compreender que principais indicadores altmétricos levantados nesta pesquisa indicaram que a maioria das publicações possui menções em fontes on-line ao passo que o nível de atenção on-line é maior para os artigos mais recentes. Dessa forma, como já observado em outros estudos altmétricos, o Mendeley e o Twitter são as principais redes sociais de circulação de resultados de pesquisas. Complementarmente, destaca-se um número significativo de menções aos artigos em notícias, o que indica que especificamente na área da Ciência da Informação, os indicadores altmétricos podem contribuir com os estudos relacionados à divulgação científica na área. Importante destacar ainda que grande parte dos periódicos possui perfis em redes sociais, o que denota que os editores estão atentos a nova convergência da utilização das métricas alternativas para complementação dos indicadores de impacto da produção científica.

Sobre o referencial teórico levantado neste artigo observou-se que os estudos sobre as métricas alternativas geralmente possuem duas vertentes: aqueles que buscam, por meio de estudo teórico, entender a representatividade dessa abordagem quantitativa para a avaliação da produção científica e aqueles que constroem e analisam os indicadores altmétricos, utilizando fontes e ferramentas altmétricas diversas.

Refletindo-se sobre os indicadores apresentados entende-se que os periódicos da Ciência da Informação devem aumentar o impacto científico na sociedade por meio da aderência às diferentes mídias sociais, como podcasts, Youtube e jornais, de maneira a contribuir para maior divulgaçãodas pesquisas da área. Realizar parcerias com as assessorias de comunicação das instituições às quais esses periódicos estão vinculados para produção de notas de divulgação científica é uma estratégia que pode contribuir no processo de divulgação científica.

Embora esta pesquisa apresente limitações no que se refere a seleção dos periódicos para os Qualis A1 e a apresentação apenas dos dados altmétricos dos principais artigos, conclui-se que diante do cenário apresentado, há um contexto favorável para a utilização de indicadores altmétricos como recurso complementar na avaliação da produção científica, tanto como contribuição para os estudos da Comunicação Científica, como para os estudos sobre a Divulgação Científica.Como proposição de pesquisas futuras sugere-se alargar o corpus dessa pesquisa buscando caracterizar de forma exaustiva a aderência dos periódicos da Ciência da Informação no contexto dos indicadores altmétricos.

\section{Referências}

ADIE, E. ; ROE, W. Altmetric: Enriching scholarly content with article-level discussion and metrics. Learned Publishing, v. 26, n. 1, p. 11-17, jan.

2013. DOI: https://doi.org/10.1087/20130103 
ALPERIN, J. P. Exploring altmetrics in an emerging country context. In: ALTMETRICS14: EXPANDING IMPACTS AND METRICS, 2014, Bloomington. Anais... Bloomington, 2014.DOI:

http://dx.doi.org/10.6084/m9.figshare.1041797

ARAÚJO, R. et al. Top AltmetricScores in the Parkinson's Disease Literature. Journal of Parkinson's disease, Amsterdam, v. 7, n. 1, p. 8187, 2017.DOI: https://doi.org/10.3233/JPD-179000

ARAÚJO, R. F. de. Cientometria 2.0, visibilidade e citação: uma incursão altmétrica em artigos de periódicos da ciência da informação. In:

ENCONTRO BRASILEIRO DE BIBLIOMETRIA E CIENTOMETRIA, 4., 2014, Recife. Anais... Recife: UFPE, 2014. Disponível em:

http://www.brapci.inf.br/article.php?dd0 =0000014387\&dd90=dd87dc69f. Acesso em: 20 jun. 2018.

ARAÚJO, R. F. Mídias sociais e comunicação científica: análise altmétrica em artigos de periódicos da Ciência da Informação. Em Questão, Porto Alegre, v.21, n.1, jan./abr. 2015. DOI: https://doi.org/10.19132/18085245211.96-109

ASNAFI, A. R. Presence of the Iranian Library and the Information Science departments in ResearchGate. DESIDOC Journal of Library \& Information Technology, New Delhi, v. 37, n. 4, p. 259-263, 2017.DOI: https://doi.org/10.14429/djlit.37.4.10561

BORNMANN, L. Validity of altmetrics data for measuring societal impact: a study using data from Altmetric and F1000 Prime. Journal of Informetrics, Amsterdam, v. 8, n. 4, p. 935-950, 2014. DOI:

https://doi.org/10.1016/j.joi.2014.09.007

BUENO, W. C. Comunicação cientifica e divulgação científica:

aproximações e rupturas conceituaiss. Informação \& Informação, Londrina, v. 15, n. 1 esp, p. 1-12, dez. 2010. DOI:

http://dx.doi.org/10.5433/1981-8920.2010v15n1espp1

BURKE, M.; KRAUT, R. E. The relationship between facebook use and well-being depends on communication type and tie strength. Journal of Computer-mediated Communication, Hoboken, v. 21, n. 4, p. 265-281, 2016.DOI: https://doi.org/10.1111/jcc4.12162

FAUSTO, S. Altmetrics, altmétricas, altmetrias: novas perspectivas na visibilidade e no impacto das pesquisas científicas. 2013. Disponível em: http://blog.scielo.org/blog/2013/08/14/altmetrics-altmetricas-altmetriasnovas-perspectivasna-visibilidade-e-no-impacto-das-pesquisascientificas/\#.U7mOWvldWSp. Acesso em: 10 jul. 2018. 
GASPARYAN, A. Y. et al. Researcher and author profiles: opportunities, advantages, and limitations. Journal of Korean Medical Science, Seoul, v. 32 , n. 11 , p. 1749-1756, 2017.DOI:

https://doi.org/10.3346/jkms.2017.32.11.1749

HAUSTEIN, S.; COSTAS, R.; LARIVIÈRE, V. Characterizing social media metrics of scholarly papers: the effect of document properties and collaboration patterns. Public Library of Science, San Francisco, v. 10, n. 3, p. e0120495, 2015.DOI:

https://doi.org/10.1371/journal.pone.0120495

HOLMBERG, K.; VAINIO, J. Why do some research articles receive more on-line attention and higher altmetrics? Reasons for on-line success according to the authors. Scientometrics, v. 116, p. 435-447, 2018. DOI:https://doi.org/10.1007/s11192-018-2710-1

KE, Q.; AHN, Y.-Y.; SUGIMOTO, C. R. A systematic identification and analysis of scientists on Twitter. Public Library of Science, San Francisco, v. 12, n. 4, p. e0175368, 2017.DOI:

https://doi.org/10.1371/journal.pone.0175368

LUIZ, O. do C. Ciência e risco à saúde nos jornais diários. São Paulo: Annablume, 2006.

MASSOLA, G. M.; CROCHIK, J. L.; SVARTMAN, B. P. Por uma crítica da divulgação científica. Psicol. USP, São Paulo, v. 26, n. 3, p. 310-315, Dez. 2015. DOI: https://doi.org/10.1590/0103-656420152603

MELERO, R. Altmetrics: a complement to conventional metrics. Biochem. Med., Zagreb, v. 25, 152-60, 2015. DOI:

https://doi.org/10.11613/BM.2015.016

MOHAMMADI, E.; THELWALL, M. Mendeley readership altmetrics for the social sciences and humanities: research evaluation and knowledge flows. Journal of the Association for Information Science and Technology, Hoboken, v. 65, p. 1627-1638, 2014.DOI:

https://doi.org/10.1002/asi. 23071

NASCIMENTO, A. G. do; ODDONE, N. Uso de indicadores altmetrics na avaliação de periódicos científicos brasileiros em Ciência da Informação. In: ENCONTRO BRASILEIRO DE BIBLIOMETRIA E CIENTOMETRIA, 4. , 2014, Recife. Anais...Recife: UFPE, 2014. DOI: https://dx.doi.org/10.6084/m9.figshare.1402366.v1 
NASSI-CALÒ, L. Métricas de avaliação em ciência: estado atual e perspectivas [Editorial]. Rev. Latino-Am. Enfermagem, Ribeirão Preto, v. 25, p. e2865, 2017. DOI: 10.1590/1518-8345.0000.2865

RAVENSCROFT, J. et al. Measuring scientific impact beyond academia: An assessment of existing impact metrics and proposed improvements. Public Library of Science, San Francisco, v. 12, n. 3, p. e0173152, 2017.DOI:https://doi.org/10.1371/journal.pone.0173152

ROBINSON-GARCIA, N. et al. The unbearable emptiness of tweeting: about journal articles. Public Library of Science, San Francisco, v. 12, n. 8, p. e0183551, 2017.DOI: https://doi.org/10.1371/journal.pone.0183551

SCHMITT, M.; JÄSCHKE, R. What do computer scientists tweet? Analyzing the link-sharing practice on Twitter. Public Library of Science, San Francisco, v. 12, n. 6, p. e0179630, 2017.DOI: https://doi.org/10.1371/journal.pone.0179630

SILVA, M. R. da. Crédito científico e métricas alternativas: possíveis aproximações. In: LUCAS, E. R. O.; SILVEIRA, M. A. (Orgs). A Ciência da Informação encontra Bourdieu. Recife: Ed. UFPE, 2017. p. 129.

SOUZA, U. J. et al. O uso das redes sociais pelos periódicos brasileiros de biblioteconomia e ciência da informação. Revista ACB: Biblioteconomia em Santa Catarina, Florianópolis, v. 20, n. 3, p. 584-591, set./dez., 2015.

THELWALL, M. et al. Do altmetrics work? Twitter and ten other social web services. Public Library of Science, San Francisco, v. 8, n. 5, p. e64841, 2013. DOI: https://doi.org/10.1371/journal.pone.0064841

TORRES-SALINAS, D.; CABEZAS-CLAVIJO, A.; JIMÉNEZ-CONTRERAS, E. Altmetrics: new indicators for scientific communication in web 2.0. Comunicar, v. 41, p. 53-60, 2013. DOI: https://doi.org/10.3916/C412013-05

WALTMAN, L.; COSTAS, R.F1000 recommendations as a potential new data source for research evaluation: A comparison with citations. Journal of the Association for Information Science and Technology, Hoboken, v. 65, n.3, p. 433-445, 2014.DOI: https://doi.org/10.1002/asi.23040 